\title{
Variabilidade espacial do solo e da biomassa epígea de pastagem, identificada por meio de geostatística
}

\author{
Célia Regina Grego(1), Cristina Aparecida Gonçalves Rodrigues(1), Sandra Furlan Nogueira(1), \\ Flávia Maria Andrade Gimenes $^{(2)}$, Alex de Oliveira( ${ }^{(3)}$, Caio Gusmão Ferrer de Almeida ${ }^{(1)}$, \\ André Luiz dos Santos Furtado(1) e João José Assumpção de Abreu Demarchi(2)
}

\begin{abstract}
(1)Embrapa Monitoramento por Satélite, Avenida Soldado Passarinho, no 303, Fazenda Chapadão, CEP 13070-115 Campinas, SP. E-mail: crgrego@cnpm.embrapa.br, crisagr@cnpm.embrapa.br, sandra@cnpm.embrapa.br, caio@cnpm.embrapa.br, andre@cnpm.embrapa.br (2)Instituto de Zootecnia, Rua Heitor Penteado, no 56, Centro, CEP 13460-000 Nova Odessa, SP. E-mail: fmeandrade@iz.sp.gov.br, demarchi@iz.sp.gov.br ${ }^{(3)}$ Anhanguera Educacional, Avenida Eugênio Biancalana Duarte, no 501, Jardim Primavera, CEP $13173-050$ Sumaré, SP. E-mail: alex.oliveira@aesapar.com
\end{abstract}

Resumo - O objetivo deste trabalho foi identificar a estrutura de dependência espacial de atributos do solo e sua interferência na produção de biomassa epígea de pastagem de capim-marandu (Urochloa brizantha). Amostras de Latossolo Vermelho-Amarelo foram coletadas em rede de pontos georreferenciados, para a determinação de atributos físicos e químicos do solo e da produção de biomassa epígea da pastagem, no verão e outono de 2010/2011. Verificou-se dependência espacial das variáveis, por meio de análise geoestatística com construção e ajuste de semivariogramas, interpolação por krigagem ordinária e espacialização em mapas de isolinhas. A dependência espacial ocorreu para alguns atributos físicos e químicos do solo (areia, densidade, resistência à penetração, infiltração, pH, MO, $\mathrm{P}, \mathrm{K}, \mathrm{Ca}, \mathrm{Mg}, \mathrm{H}+\mathrm{Al}, \mathrm{Al}, \mathrm{CTC}$ e $\mathrm{V}$ ), e para a biomassa da pastagem medida nas estações verão e outono. A análise espacial de atributos físicos do solo permite identificar áreas degradadas da pastagem. A biomassa da pastagem é mais influenciada pelos atributos físicos do solo do que pelos químicos.

Termos para indexação: Urochloa brizantha, atributos do solo, dependência espacial, espacialização.

\section{Variability of soil and pasture epigeal biomass identified by geostatistics}

\begin{abstract}
The objective of this work was to identify the spatial structure dependence of soil attributes and its interference on the production of epigeal biomass of Urochloa brizantha. A Typic Hapludox was sampled at geocoded grid points in order to determine soil physical and chemical properties and the epigeal biomass production of pasture during the summer and fall seasons of 2010/2011. Spatial dependence of variables was verified by geostatistical analysis using construction and adjustment of semivariograms, ordinary kriging interpolation, and spatial maps. Spatial dependence occurred for some physical and chemical soil properties (sand, density, penetration resistance, infiltration, $\mathrm{pH}, \mathrm{MO}, \mathrm{P}, \mathrm{K}, \mathrm{Ca}, \mathrm{Mg}, \mathrm{H}+\mathrm{Al}, \mathrm{Al}, \mathrm{CEC}$, and base saturation), and for pasture biomass measured in the summer and fall seasons. Spatial analysis of soil physical attributes allows identifying areas with the highest pasture degradation. Biomass pasture is more influenced by the soil physical attributes than by the chemical ones.
\end{abstract}

Index terms: Urochloa brizantha, soil attributes, spatial dependence, spatialization.

\section{Introdução}

O Estado de São Paulo tem aproximadamente 8,1 milhões de hectares ocupados com pastagem, dos quais a maior parte $(61,7 \%)$ é utilizada com plantas do gênero Urochloa (Syn. Brachiaria) (São Paulo, 2008), de elevada rusticidade e adaptação a solos ácidos e pouco férteis (Bonfim et al., 2003).

O conhecimento da distribuição espacial dos atributos do solo e de suas relações com a pastagem pode auxiliar no seu manejo. As causas da degradação de pastagens variam e incluem: práticas inadequadas de pastejo e manejo, falhas no estabelecimento e fatores bióticos (pragas e doenças) e abióticos (excesso ou falta de chuvas, baixa fertilidade e drenagem insuficiente dos solos) (Dias-Filho, 2011).

O manejo de animais sobre as pastagens causa modificações físicas do solo em médio e longo prazo, e estas modificações variam no espaço. Miguel et al. (2009) relataram a dependência espacial de atributos do solo em pastagens e observaram variação na infiltração de água em função da intensidade de pisoteio.

Atributos de solo normalmente apresentam forte dependência espacial, uma vez que variam conforme o

Pesq. agropec. bras., Brasília, v.47, n.9, p.1404-1412, set. 2012 
local na paisagem (Vieira \& Dechen, 2010; Grego et al., 2011; Vidal-Vázquez et al., 2012). A geoestatistica é uma ferramenta adequada e fundamental para a análise de propriedades variáveis no espaço, que apresentam algum grau de organização ou continuidade detectáveis por medidas de dependência espacial (Vieira \& Dechen, 2010). Nesse sentido, Souza et al. (2006) mostraram que há mais variabilidade em pedoformas côncavas e convexas de Latossolos do que em pedoformas lineares, independentemente do histórico de manejo. Ceddia et al. (2009) analisaram a variabilidade de atributos físicos do solo conforme a diferença de altitude, e verificaram que a dependência espacial decresceu com o aumento da profundidade amostrada do solo.

Ao se considerar que a condição física e química do solo afeta diretamente o desenvolvimento das plantas, as investigações da variabilidade espacial do solo, juntamente com a da biomassa produzida pela forrageira, ao longo do tempo, podem subsidiar decisões quanto ao planejamento e ao manejo de áreas com pastagem. Portanto, a análise geoestatística aplicada a essas áreas pode ser importante para identificar padrões espaciais de solo e de planta que auxiliem na otimização do sistema de produção.

O objetivo deste trabalho foi identificar a estrutura de dependência espacial dos atributos do solo e sua interferência na produção de biomassa epígea de pastagem de capim-marandu (Urochloa brizantha Hochst. ex A. Rich).

\section{Material e Métodos}

A área estudada, de 3,67 ha, localiza-se na Fazenda Experimental do Instituto de Zootecnia, Nova Odessa, SP (22 $46^{\prime} 39^{\prime \prime} \mathrm{S}$ e $47^{\circ} 17^{\prime} 45^{\prime \prime} \mathrm{W}$, a $570 \mathrm{~m}$ de altitude), em um Latossolo Vermelho-Amarelo sob pastagem degradada de $U$. brizantha, utilizada para reserva de animais no pastejo rotacionados de piquetes próximos.

Para a amostragem, utilizou-se uma rede de 50 pontos georreferenciados, distanciados entre si de, aproximadamente $30 \mathrm{~m}$, que não mostraram regularidade de espaçamento, em razão do erro de medição das coordenadas pelo aparelho GPS Garmim (Figura 1). A área é representativa de pastagens sem aplicação de práticas de manejo, no Estado de São Paulo.

Quanto aos atributos físicos do solo, foram avaliados: granulometria, tendo-se determinado os teores de areia, silte e argila na profundidade de $0-0,20 \mathrm{~m}$; densidade global, determinada em amostras indeformadas, conforme Camargo et al. (1986), nas profundidades de $0-0,10$ e de $0,10-0,20 \mathrm{~m}$; resistência à penetração, medida com penetrômetro de impacto, segundo Stolf (1991), na camada de $0-0,30 \mathrm{~m}$, com intervalos de 0,05 m; permeabilidade, avaliada com permeâmetro, modelo IAC, de acordo com método estabelecido por Vieira (1998); e infiltração tridimensional do solo a $0,20 \mathrm{~m}$.

Para a análise dos atributos químicos, foram coletadas amostras nas profundidades de $0-0,10$ e $0,10-0,20 \mathrm{~m}$. Os atributos químicos analisados foram: $\mathrm{pH}$ em $\mathrm{H}_{2} \mathrm{O}$, $\mathrm{K}, \mathrm{Ca}, \mathrm{Mg}, \mathrm{Al}, \mathrm{P}$ (Mehlich 1), matéria orgânica, soma de bases, acidez potencial, CTC, saturação por bases e saturação por alumínio, conforme Claessen (1997).

Dados da biomassa epígea da pastagem foram estimados de acordo com o método do rendimento comparativo (Haydock \& Shaw, 1975). Quadrados de ferro, com área de $0,25 \mathrm{~m}^{2}$, foram marcados na pastagem, na mesma rede de amostragem (Figura 1). Foram atribuídos escores de 1 a 5, por três observadores, em que 1 representa a menor quantidade de forragem disponível dentro da pastagem e 5, a maior. A atribuição de escores foi feita por meio de avaliações visuais de altura e densidade.

Em seguida, o material vegetal contido dentro dos quadrados foi coletado e seco em estufa, tendo-se realizado a análise de regressão entre o escore visual e o peso da forragem seca. Essas análises foram realizadas a cada 28 dias, durante oito meses, sempre nos mesmos locais de amostragem, e os valores de biomassa epígea foram agrupados em $\mathrm{kg} \mathrm{ha}^{-1}$ de matéria seca, durante os meses de verão (novembro e dezembro de 2010, e janeiro e fevereiro de 2011) e de outono (março, abril, maio e junho de 2011).

Os dados foram analisados por meio de estatística descritiva, para exploração inicial e verificação da normalidade da distribuição de frequência dos dados. Para distribuições normais de frequência, os valores de assimetria e curtose foram próximos de zero.

Para verificar a dependência espacial das variáveis, interpolar dados e elaborar os mapas, empregou-se a análise geoestatística segundo Vieira (2000). Foram construídos semivariogramas, a partir das pressuposições de estacionariedade da hipótese intrínseca e do cálculo da semivariância, estimada pela equação: 
$\gamma(\mathrm{h})=\frac{1}{2 \mathrm{~N}(\mathrm{~h})} \sum_{\mathrm{i}=1}^{\mathrm{N}}\left[\mathrm{Z}\left(\mathrm{x}_{\mathrm{i}}\right)-\mathrm{Z}\left(\mathrm{x}_{\mathrm{i}}+\mathrm{h}\right)\right]^{2}$

em que N(h) é o número de pares dos valores medidos $\mathrm{Z}\left({ }_{\mathrm{xi}}\right)$ e $\mathrm{Z}(\mathrm{xi+h})$, separados por um vetor $\mathrm{h}$.

Medições realizadas em locais próximos são mais parecidas entre si do que as separadas por grandes distâncias, isto é, há aumento de $\gamma(\mathrm{h})$ com a distância $\mathrm{h}$ até um valor máximo, no qual se estabiliza em um patamar correspondente à distância limite de dependência espacial, que é o alcance (Vieira, 2000). Medições localizadas a distâncias maiores que o alcance têm distribuição aleatória e, portanto, são independentes entre si.

Os semivariogramas foram ajustados ao modelo matemático de melhor correspondência. Após ajuste destes, pôde-se estimar valores para os locais não amostrados, com condições de não tendenciosidade e variância mínima, por meio da krigagem ordinária.

Os programas computacionais, os procedimentos para construção e ajuste do modelo do semivariograma e a krigagem foram baseados em Vieira et al. (2002).

\section{Resultados e Discussão}

O solo apresentou, na camada até $0,20 \mathrm{~m}$, textura areno-argilosa, densidade média a elevada, baixa infiltração de água e valores de resistência à penetração relativamente altas (Tabela 1). Os resultados de resistência à penetração indicam que o solo da área de estudo encontra-se em condição de degradação. $\mathrm{O}$ manejo inadequado de animais sobre as pastagens deteriora os atributos físicos do solo (Lanzanova et al., 2007; Miguel et al., 2009). O efeito compactante do

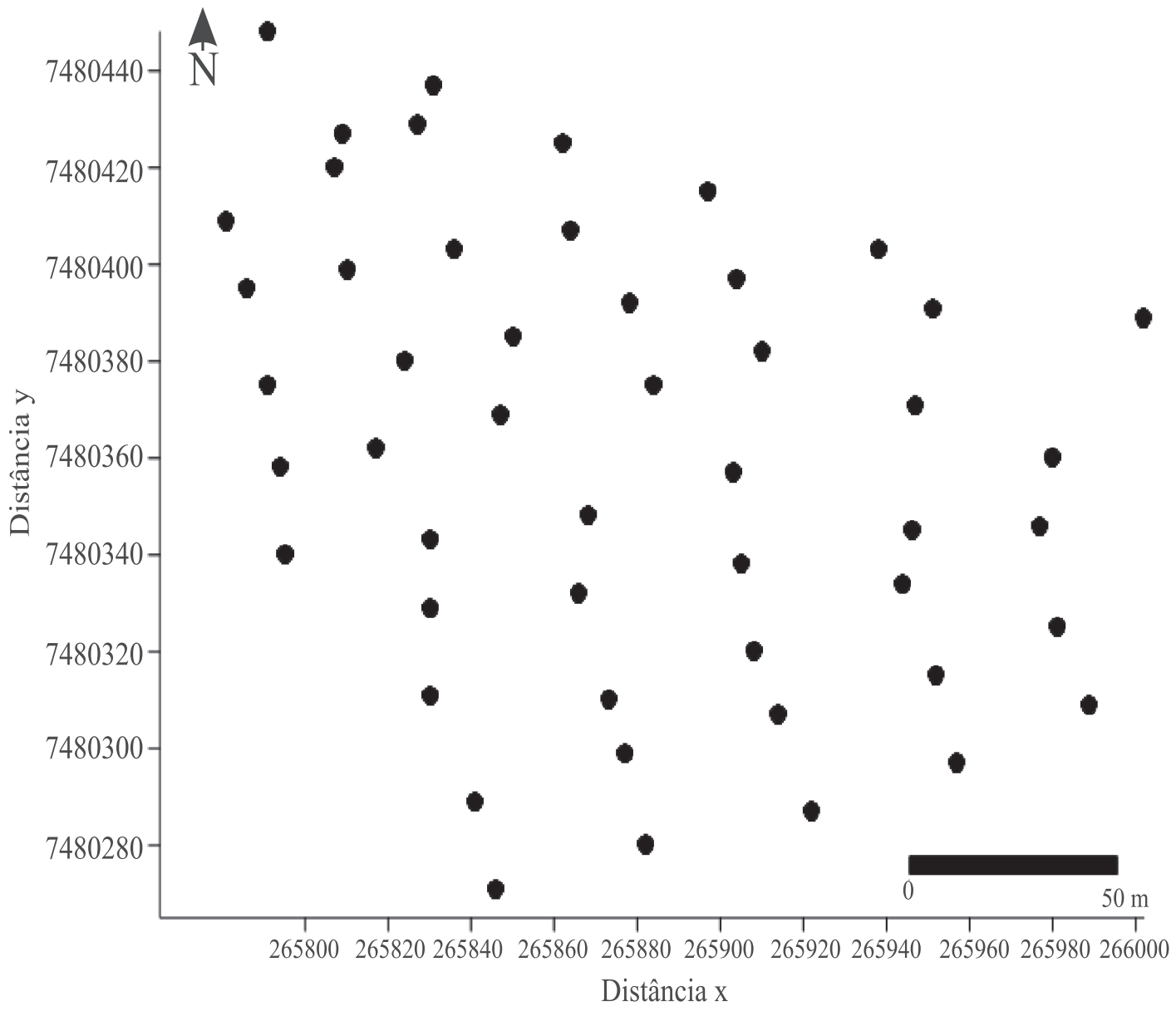

Figura 1. Rede de amostragem de solo com 50 pontos georreferenciados (coordenadas em UTM) na área de estudo com pastagem de Urochloa brizantha. 
pisoteio animal, na camada superficial do solo, pode resultar em alteração da estrutura, selamento superficial e compactação (Pires et al., 2012).

Os atributos químicos do solo apresentaram distribuição normal, mas com valores relativamente altos de assimetria e curtose, e indicaram baixa fertilidade do solo (Tabela 1). A produção de biomassa epígea foi maior no outono do que no verão, devido o período seco em que as coletas no verão foram realizadas. Mesmo com essa limitação da amostragem, a produção de biomassa no verão não foi considerada baixa. Santos et al. (2007) encontraram valor médio bem menor para produção da parte aérea $\left(1.280 \mathrm{~kg} \mathrm{ha}^{-1}\right)$, em pastagens de $U$. brizantha 'Marandu', sob estágio moderado de degradação, no período chuvoso, de dezembro a março.

Todos os dados submetidos à análise estatística descritiva, também foram analisados geoestatistica-mente, porém nem todos apresentaram dependência espacial. Pelo ajuste do semivariograma aos dados com dependência espacial, foram obtidos o efeito pepita(Co), a variância estrutural (C1) e o alcance

Tabela 1. Média, variância, desvio-padrão, coeficiente de variação (CV), valor mínimo, valor máximo, assimetria e curtose, para atributos físicos e químicos do solo, bem como para biomassa epígea.

\begin{tabular}{|c|c|c|c|c|c|c|c|c|}
\hline Variáveis & Média & Variância & Desvio-padrão & $\mathrm{CV}(\%)$ & Mínimo & Máximo & Assimetria & Curtose \\
\hline & \multicolumn{8}{|c|}{ Atributos físicos } \\
\hline Areia $0-0,20 \mathrm{~m}\left(\mathrm{~g} \mathrm{~kg}^{-1}\right)$ & 364,90 & $4.174,00$ & 64,60 & 17,71 & 230,00 & 459,00 & $-0,38$ & $-0,80$ \\
\hline Argila $0-0,20 \mathrm{~m}\left(\mathrm{~g} \mathrm{~kg}^{-1}\right)$ & 367,70 & $4.391,00$ & 66,26 & 18,02 & 253,00 & 470,00 & $-0,21$ & $-1,00$ \\
\hline Silte $0-0,20 \mathrm{~m}\left(\mathrm{~g} \mathrm{~kg}^{-1}\right)$ & 267,50 & $4.064,00$ & 63,75 & 23,83 & 148,00 & 373,00 & $-0,12$ & $-0,92$ \\
\hline Densidade $0-0,10 \mathrm{~m}\left(\mathrm{~g} \mathrm{~cm}^{-3}\right)$ & 1,44 & 0,02 & 0,13 & 8,95 & 1,16 & 1,65 & $-0,27$ & $-0,32$ \\
\hline Densidade $0,10-0,20 \mathrm{~m}\left(\mathrm{~g} \mathrm{~cm}^{-3}\right)$ & 1,46 & 0,02 & 0,12 & 8,47 & 1,03 & 1,67 & $-1,17$ & 4,52 \\
\hline Infiltração $0,20 \mathrm{~m}_{\left(\mathrm{mm} \mathrm{h}^{-1}\right)}$ & 18,69 & 231,60 & 15,22 & 81,44 & 6,04 & 96,59 & 3,17 & 14,38 \\
\hline RP 0-0,05 m (MPa) & 4,71 & 5,47 & 2,34 & 49,72 & 0,55 & 14,07 & 1,26 & 3,96 \\
\hline $\mathrm{RP} 0,05-0,10 \mathrm{~m}(\mathrm{MPa})$ & 5,31 & 2,94 & 1,71 & 32,28 & 2,48 & 10,69 & 1,14 & 1,60 \\
\hline $\mathrm{RP} 0,10-0,15 \mathrm{~m}(\mathrm{MPa})$ & 6,31 & 4,72 & 2,17 & 34,44 & 2,48 & 13,40 & 0,97 & 1,89 \\
\hline $\mathrm{RP} 0,15-0,20 \mathrm{~m}(\mathrm{MPa})$ & 6,68 & 4,34 & 2,08 & 31,17 & 2,48 & 12,05 & 0,33 & 0,21 \\
\hline $\mathrm{RP} 0,20-0,25 \mathrm{~m}(\mathrm{MPa})$ & 7,41 & 7,32 & 2,71 & 36,54 & 2,48 & 14,75 & 0,59 & 0,20 \\
\hline \multirow[t]{2}{*}{$\underline{\mathrm{RP}} 0,25-0,30 \mathrm{~m}(\mathrm{MPa})$} & 6,95 & 9,82 & 3,13 & 45,11 & 2,48 & 16,78 & 1,35 & 1,75 \\
\hline & \multicolumn{8}{|c|}{ Atributos químicos } \\
\hline $\mathrm{pH} 0-0,1 \mathrm{~m}$ & 5,22 & 0,06 & 0,26 & 4,90 & 4,90 & 6,00 & 1,24 & 1,97 \\
\hline $\mathrm{pH} 0,1-0,2 \mathrm{~m}$ & 5,10 & 0,05 & 0,22 & 4,36 & 4,70 & 5,70 & 1,18 & 1,94 \\
\hline MO $0-0,1 \mathrm{~m}\left(\mathrm{~g} \mathrm{dm}^{-3}\right)$ & 33,32 & 21,93 & 4,68 & 14,05 & 23,00 & 43,00 & 0,23 & 0,10 \\
\hline $\mathrm{MO} 0,1-0,2 \mathrm{~m}\left(\mathrm{~g} \mathrm{dm}^{-3}\right)$ & 29,39 & 34,03 & 5,83 & 19,85 & 20,00 & 49,00 & 1,37 & 3,69 \\
\hline $\mathrm{P} 0-0,1 \mathrm{~m}\left(\mathrm{mg} \mathrm{dm}^{-3}\right)$ & 2,14 & 2,50 & 1,58 & 73,75 & 1,00 & 6,00 & 1,32 & 0,80 \\
\hline $\mathrm{P} 0,1-0,2 \mathrm{~m}\left(\mathrm{mg} \mathrm{dm}^{-3}\right)$ & 1,43 & 1,22 & 1,10 & 77,22 & 1,00 & 6,00 & 3,31 & 11,68 \\
\hline $\mathrm{K} 0-0,1 \mathrm{~m}\left(\mathrm{mmol}_{\mathrm{c}} \mathrm{dm}^{-3}\right)$ & 0,73 & 0,10 & 0,31 & 43,07 & 0,40 & 1,50 & 0,93 & $-0,24$ \\
\hline $\mathrm{K} 0,1-0,2 \mathrm{~m}\left(\mathrm{mmol}_{\mathrm{c}} \mathrm{dm}^{-3}\right)$ & 0,40 & 0,03 & 0,17 & 42,49 & 0,20 & 0,80 & 0,58 & $-0,27$ \\
\hline $\mathrm{Ca} 0-0,1 \mathrm{~m}\left(\mathrm{mmol}_{\mathrm{c}} \mathrm{dm}^{-3} \mathrm{~m}\right)$ & 12,75 & 23,68 & 4,87 & 38,16 & 7,00 & 26,00 & 1,07 & 0,99 \\
\hline $\mathrm{Ca} 0,1-0,2 \mathrm{~m}\left(\mathrm{mmol}_{\mathrm{c}} \mathrm{dm}^{-3}\right)$ & 11,14 & 23,90 & 4,89 & 43,88 & 4,00 & 23,00 & 1,08 & 0,60 \\
\hline $\operatorname{Mg} 0-0,1 \mathrm{~m}\left(\mathrm{mmol}_{\mathrm{c}} \mathrm{dm}^{-3}\right)$ & 7,32 & 6,30 & 2,51 & 34,28 & 4,00 & 14,00 & 0,94 & 0,52 \\
\hline $\operatorname{Mg} 0,1-0,2 \mathrm{~m}\left(\mathrm{mmol}_{\mathrm{c}} \mathrm{dm}^{-3}\right)$ & 5,93 & 5,48 & 2,34 & 39,47 & 2,00 & 12,00 & 0,99 & 0,85 \\
\hline $\mathrm{H}+\mathrm{Al} 0-0,1 \mathrm{~m}\left(\mathrm{mmol}_{\mathrm{c}} \mathrm{dm}^{-3}\right)$ & 43,18 & 109,30 & 10,45 & 24,21 & 24,00 & 72,00 & 1,36 & 2,51 \\
\hline $\mathrm{H}+\mathrm{Al} 0,1-0,2 \mathrm{~m}\left(\mathrm{mmol}_{\mathrm{c}} \mathrm{dm}^{-3}\right)$ & 47,96 & 163,40 & 12,78 & 26,65 & 30,00 & 88,00 & 1,40 & 2,59 \\
\hline $\mathrm{Al} 0-0,1 \mathrm{~m}\left(\mathrm{mmol}_{\mathrm{c}} \mathrm{dm}^{-3}\right)$ & 10,54 & 32,41 & 5,69 & 54,03 & 1,00 & 20,00 & 0,34 & $-0,93$ \\
\hline Al 0,1-0,2 $\mathrm{m}\left(\mathrm{mmol}_{\mathrm{c}} \mathrm{dm}^{-3}\right)$ & 14,14 & 49,39 & 7,03 & 49,69 & 1,00 & 32,00 & 0,29 & 0,42 \\
\hline CTC $0-0,1 \mathrm{~m}\left(\mathrm{mmol}_{\mathrm{c}} \mathrm{dm}^{-3}\right)$ & 64,14 & 106,80 & 10,33 & 16,11 & 55,00 & 93,00 & 1,51 & 1,92 \\
\hline CTC $0,1-0,2 \mathrm{~m}\left(\mathrm{mmol}_{\mathrm{c}} \mathrm{dm}^{-3}\right)$ & 65,43 & 151,70 & 12,32 & 18,82 & 51,00 & 101,00 & 1,69 & 2,81 \\
\hline V $0-0,1 \mathrm{~m}(\%)$ & 32,57 & 107,80 & 10,38 & 31,88 & 18,00 & 58,00 & 0,89 & 0,57 \\
\hline \multirow[t]{2}{*}{$\underline{\mathrm{V} 0,1-0,2 \mathrm{~m}(\%)}$} & 27,04 & 105,60 & 10,28 & 38,01 & 10,00 & 53,00 & 0,73 & 0,58 \\
\hline & \multicolumn{8}{|c|}{ Biomassa da pastagem $\left(\mathrm{kg} \mathrm{ha}^{-1}\right.$ de matéria seca) } \\
\hline Verão & 2.142 & 236.4 & 486,2 & 22,7 & 1.200 & 2.930 & $-1,0$ & $-0,2$ \\
\hline Outono & 6.048 & 12.260 & 3.501 & 57,9 & 2.202 & 13.710 & 0,8 & $-0,5$ \\
\hline
\end{tabular}

RP, resistência à penetração; $\mathrm{MO}$, matéria orgânica do solo; $\mathrm{V}(\%)$, saturação por bases. 
(a) (Tabelas 2 e 3). Para os dados que não apresentaram dependência espacial, não foi possível o ajuste dos semivariogramas, pela inexistência de patamar. $\mathrm{O}$ número reduzido de amostras pode ter causado a inexistência de dependência para alguns dados, pois as amostragens devem ser suficientemente próximas para que se possa caracterizar possíveis manchas de variabilidade. Portanto, distância de amostragem menores do que os obtidos neste trabalho poderiam melhorar a precisão no ajuste dos semivariogramas (Silva Neto et al., 2012). Atualmente, tem-se utilizado variáveis indicadoras para otimizar a amostragem, tais como dados obtidos por sensores, imagem de satélite e fotos aéreas (Vieira et al., 2008). No presente trabalho, a análise geoestatística poderia ter sido beneficiada pelo uso de imagens de satélite de alta resolução espacial, as quais identificariam maior necessidade de pontos amostrais em regiões com maior variabilidade.

Foi observada dependência espacial para os atributos físicos areia, densidade, infiltração de água e resistência à penetração (Tabela 2). Para a maioria dos atributos químicos do solo e para biomassa da pastagem, houve dependência espacial com predomínio de ajuste dos semivariogramas pelo modelo esférico (Tabela 3 ).

Os mapas (Figuras 2, 3 e 4) permitiram inferir que a porção superior esquerda da área apresentou maior teor de areia, densidade do solo, resistência à penetração e menor infiltração de água (Figura 2). De acordo com Tavares Filho \& Ribon (2008), o teor de argila apresenta correlação positiva com o grau

Tabela 2. Parâmetros de ajuste dos semivariogramas efeito pepita (Co), variância estrutural (C1) e alcance (a), coeficiente de correlação (r), grau de dependência espacial (GD) e modelo de ajuste para os atributos físicos que apresentaram dependência espacial.

\begin{tabular}{lccccccc}
\hline Variável $^{(1)}$ & $\begin{array}{c}\text { Camada } \\
(\mathrm{m})\end{array}$ & Co & $\mathrm{C} 1$ & $\begin{array}{c}\mathrm{a} \\
(\mathrm{m})\end{array}$ & $\mathrm{r}$ & $\begin{array}{c}\text { GD } \\
(\%)\end{array}$ & $\begin{array}{c}\text { Modelo } \\
\text { de ajuste }\end{array}$ \\
\hline Areia & $0-0,20$ & 2.200 & 2.350 & 110,00 & $-0,09$ & 52 & Esférico \\
Ds & $0-0,10$ & 0,00 & 0,01 & 50,00 & 0,31 & 88 & Esférico \\
Infiltração & 0,20 & 130,00 & 160,00 & 110,00 & 0,69 & 55 & Esférico \\
RP & $0,05-0,10$ & 0,10 & 3,10 & 70,00 & 0,48 & 97 & Exponencial \\
RP & $0,15-0,20$ & 1,00 & 3,40 & 85,00 & 0,44 & 77 & Esférico \\
RP & $0,20-0,25$ & 3,00 & 4,70 & 35,00 & 0,36 & 61 & Esférico \\
RP & $0,25-0,30$ & 4,80 & 4,40 & 90,00 & 0,84 & 48 & Esférico \\
\hline
\end{tabular}

${ }^{(1)} \mathrm{Ds}$, densidade do solo; RP, resistência a penetração. de compactação do solo. No entanto, para o presente trabalho, a compactação não teve relação com textura do solo, tendo sido afetada, primordialmente, por fatores externos, como o excesso de pisoteio.

Em geral, a dependência espacial da resistência do solo à penetração é difícil de ser detectada, por ser uma medida muito pontual. Nesse sentido, a dependência observada ocorreu apenas para as profundidades de $0,05-0,10,0,15-0,20,0,20-0,25$ e $0,25-0,30 \mathrm{~m}$ (Figura 2). Moreira et al. (2012) observaram que, entre os atributos físicos avaliados, a resistência à penetração foi o que apresentou maior sensibilidade na detecção de alterações físicas do solo.

Com relação aos atributos químicos, a rede amostrada não permitiu identificar forte relação entre as variáveis. Alguns atributos apresentaram mapas com manchas de maiores e menores valores na mesma localização. Este comportamento pode ser visualizado nos mapas de $\mathrm{pH}, \mathrm{CTC}, \mathrm{V}$, e entre os mapas de matéria orgânica, $\mathrm{Ca}$ e $\mathrm{Mg}$ (Figura 3 e 4). Como a pastagem estudada não recebeu nenhuma adição de fertilizantes químicos ou orgânicos, as características químicas do solo podem

Tabela 3. Parâmetros de ajuste dos semivariogramas efeito pepita (Co), variância estrutural (C1) e alcance (a), coeficiente de correlação (r), grau de dependência espacial (GD) e modelo de ajuste para os atributos químicos do solo e biomassa da pastagem que apresentaram dependência espacial.

\begin{tabular}{|c|c|c|c|c|c|c|c|}
\hline Variável $^{(1)}$ & $\begin{array}{c}\text { Camada } \\
(\mathrm{m})\end{array}$ & $\mathrm{Co}$ & $\mathrm{C} 1$ & $\begin{array}{c}\mathrm{a} \\
(\mathrm{m})\end{array}$ & $\mathrm{r}$ & $\begin{array}{l}\text { GD } \\
(\%)\end{array}$ & $\begin{array}{l}\text { Modelo } \\
\text { de ajuste }\end{array}$ \\
\hline \multicolumn{8}{|c|}{ Atributos químicos do solo } \\
\hline $\mathrm{pH}$ & $0-0,1$ & 0,01 & 0,07 & 100,0 & 0,42 & 93 & Esférico \\
\hline $\mathrm{pH}$ & $0,1-0,2$ & 0,01 & 0,04 & 80,0 & 0,23 & 80 & Esférico \\
\hline MO & $0-0,1$ & 4,00 & 17,00 & 55,0 & 0,64 & 81 & Esférico \\
\hline MO & $0,1-0,2$ & 0,00 & 30,00 & 75,0 & 0,36 & 100 & Esférico \\
\hline $\mathrm{P}$ & $0-0,1$ & 1,40 & 1,20 & 80,0 & 0,68 & 46 & Esférico \\
\hline $\mathrm{K}$ & $0,1-0,2$ & 0,01 & 0,03 & 140,0 & 0,92 & 74 & Esférico \\
\hline $\mathrm{Ca}$ & $0-0,1$ & 0,00 & 25,00 & 70,0 & $-0,37$ & 100 & Gaussiano \\
\hline $\mathrm{Mg}$ & $0-0,1$ & 0,00 & 7,50 & 85,0 & $-0,75$ & 100 & Gaussiano \\
\hline $\mathrm{Mg}$ & $0,1-0,2$ & 1,60 & 4,30 & 65,0 & $-0,08$ & 73 & Esférico \\
\hline $\mathrm{H}+\mathrm{Al}$ & $0,1-0,2$ & 12,00 & 140,00 & 65,0 & $-0,38$ & 92 & Esférico \\
\hline $\mathrm{Al}$ & $0,1-0,2$ & 18,00 & 30,00 & 80,0 & 0,42 & 63 & Esférico \\
\hline CTC & $0,1-0,2$ & 50,00 & 100,00 & 50,0 & $-0,18$ & 67 & Esférico \\
\hline$\underline{\mathrm{V}}$ & $0-0,1$ & 10,00 & 106,00 & 90,0 & $-0,34$ & 91 & Esférico \\
\hline \multicolumn{8}{|c|}{ Biomassa da pastagem } \\
\hline BV & - & 90000 & 120000 & 70,0 & $-0,14$ & 57 & Esférico \\
\hline$\underline{\mathrm{BO}}$ & - & 5000000 & 6000000 & 60,0 & 0,35 & 55 & Esférico \\
\hline
\end{tabular}


ter refletido a composição original do solo e a ciclagem interna de nutrientes na superfície do solo. Segundo Fisher et al. (2007) as forrageiras tropicais são capazes de sequestrar grandes quantidades de carbono no solo, os quais posteriormente são carreados para as camadas mais profundas.
Os mapas da produção de biomassa epígea evidenciam que a produção no outono foi maior do que a do verão indicando o efeito da condição climática no período amostrado (Figura 4). Ao comparar os mapas de biomassa com os dos atributos do solo, é identificada maior relação com

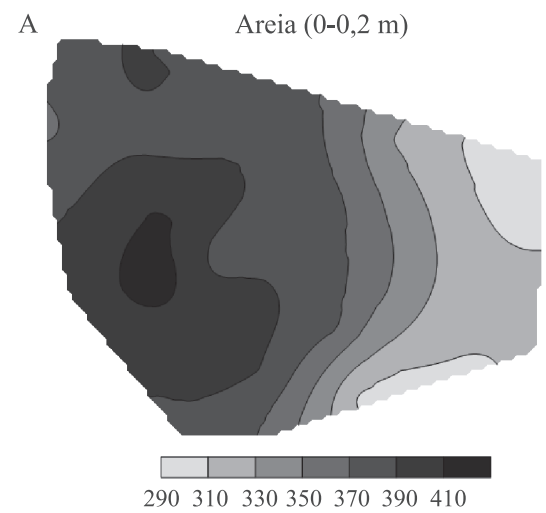

D Resistência à penetração $(0,05-0,10 \mathrm{~m})$

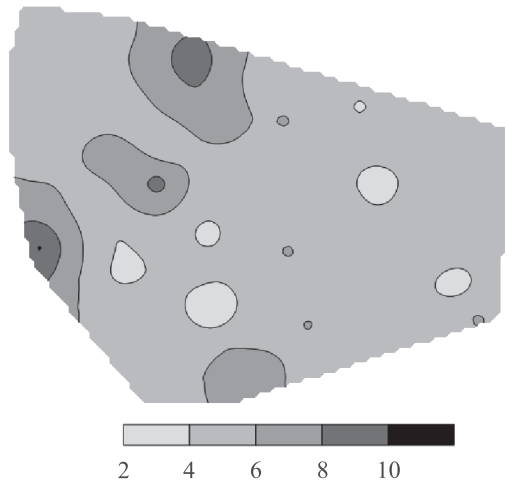

B

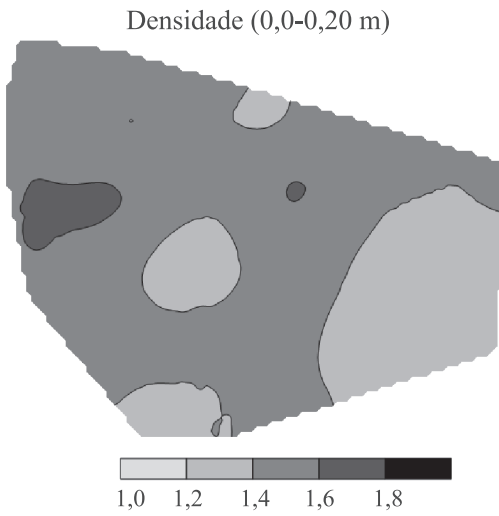

E Resistência à penetração $(0,15-0,20 \mathrm{~m})$

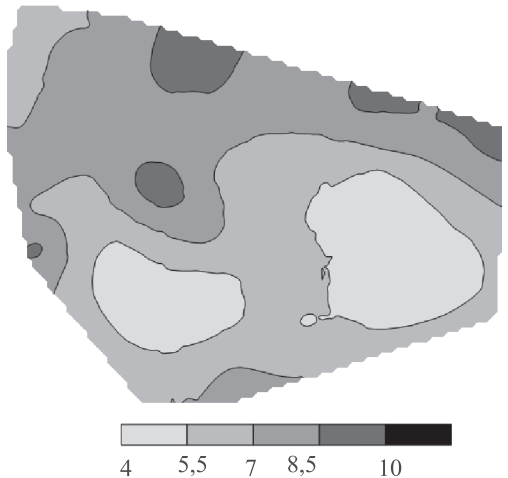

G Resistência à penetração $(0,25-0,30 \mathrm{~m})$

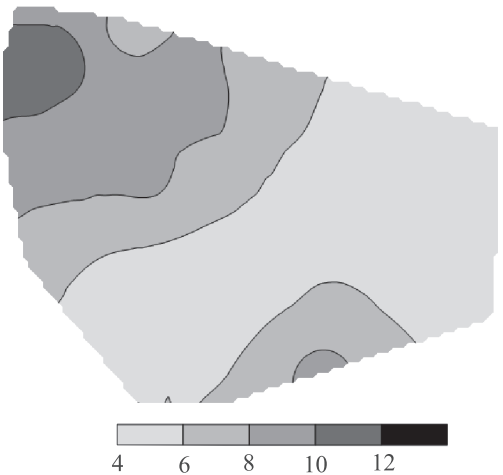

$\mathrm{C}$

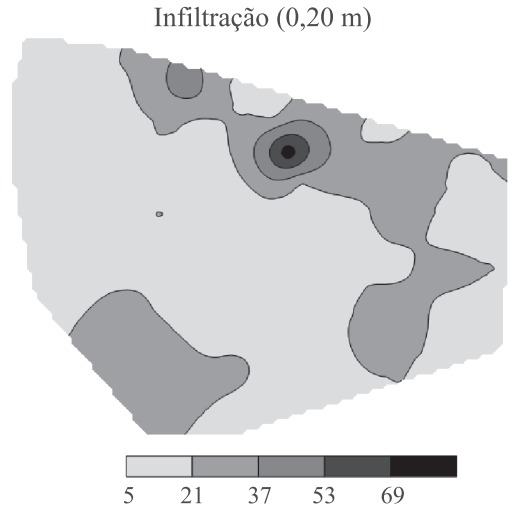

F Resistência à penetração $(0,20-0,25 \mathrm{~m})$

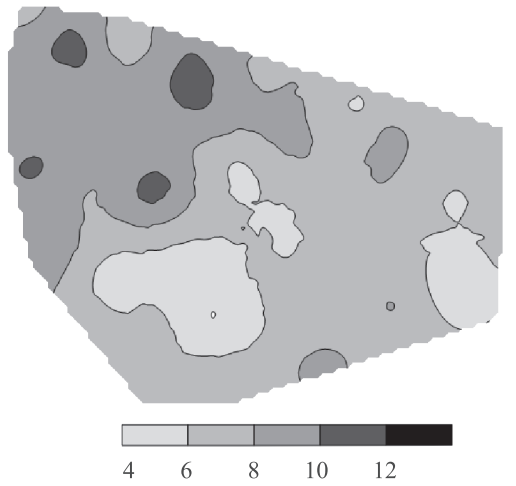

Figura 2. Mapas espacializados por krigagem ordinária dos atributos físicos do solo que apresentaram dependência espacial: $\mathrm{A}$, areia $\left(\mathrm{g} \mathrm{kg}^{-1}\right) ; \mathrm{B}$, densidade $\left(\mathrm{g} \mathrm{cm}^{-3}\right) ; \mathrm{C}$, infiltração $\left(\mathrm{mm} \mathrm{h}^{-1}\right) ; \mathrm{D}$, resistência à penetração $(\mathrm{MPa}) ; \mathrm{E}$, resistência à penetração $(\mathrm{MPa}) ; \mathrm{F}$, resistência à penetração $(\mathrm{MPa}) ; \mathrm{G}$, resistência à penetração $(\mathrm{MPa})$. 
os atributos físicos do que com os químicos do solo. Quando os mapas de biomassa são comparados entre si, as manchas de variabilidade espacial não foram correlacionadas positivamente, pois a estrutura espacial observada após o período de verão (seca) não manteve no espaço após o outono (período chuvoso). Dessa forma, foi possível observar nos mapas que, na parte direita superior da área, após as chuvas, houve crescimento da biomassa muito maior do que no restante da área.
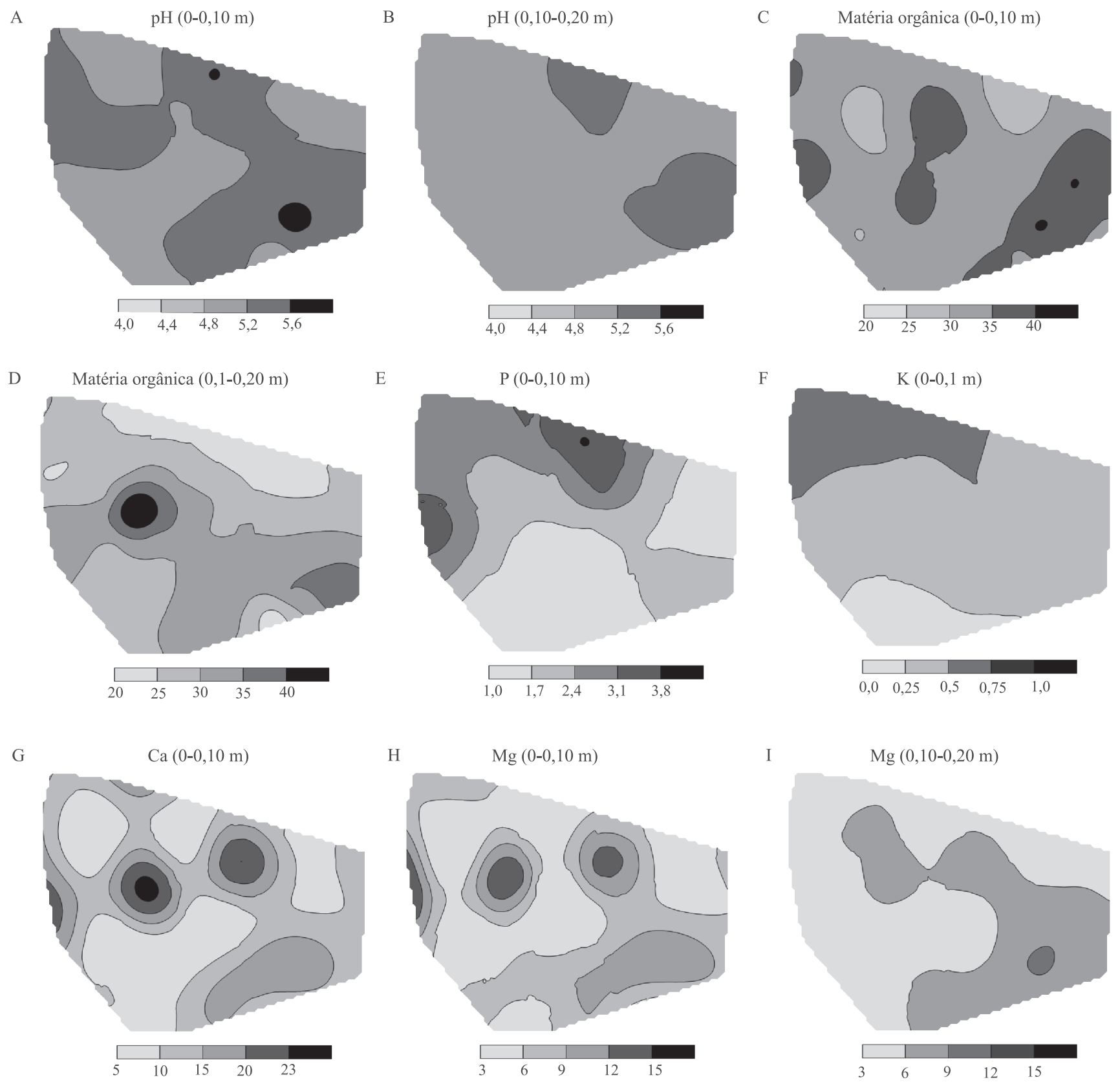

Figura 3. Mapas espacializados por krigagem ordinária dos atributos químicos do solo e da biomassa da pastagem: $\mathrm{A}, \mathrm{pH}$ em $\mathrm{H}_{2} \mathrm{O} ; \mathrm{B}, \mathrm{pH}$ em $\mathrm{H}_{2} \mathrm{O} ; \mathrm{C}, \mathrm{MO}\left(\mathrm{g} \mathrm{dm}^{-3}\right) ; \mathrm{D}, \mathrm{MO}\left(\mathrm{g} \mathrm{dm}^{-3}\right)$; E, P resina $\left(\mathrm{mg} \mathrm{dm}^{-3}\right) ; \mathrm{F}, \mathrm{K}\left(\mathrm{mmol}_{\mathrm{c}} \mathrm{dm}^{-3}\right) ; \mathrm{G}, \mathrm{Ca}\left(\mathrm{mmol}_{\mathrm{c}} \mathrm{dm}^{-3}\right) ; \mathrm{H}$, $\mathrm{Mg}\left(\mathrm{mmol}_{\mathrm{c}} \mathrm{dm}^{-3}\right) ; \mathrm{I}, \mathrm{Mg}\left(\mathrm{mmol}_{\mathrm{c}} \mathrm{dm}^{-3}\right)$. 
A

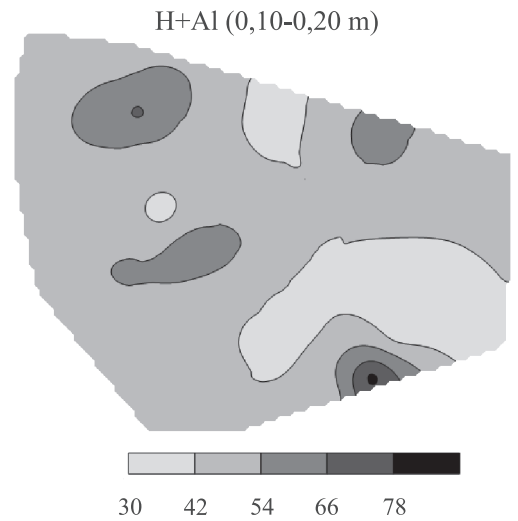

D

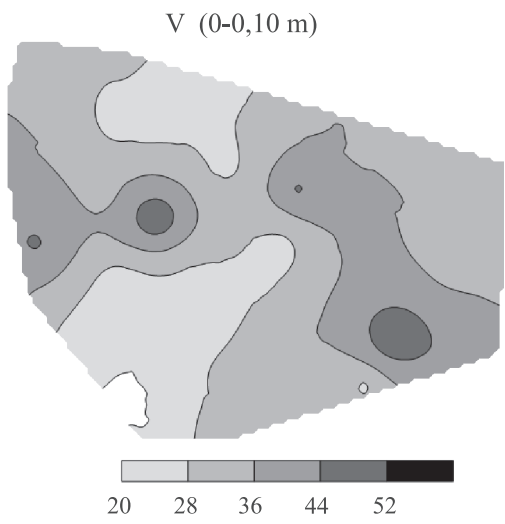

B

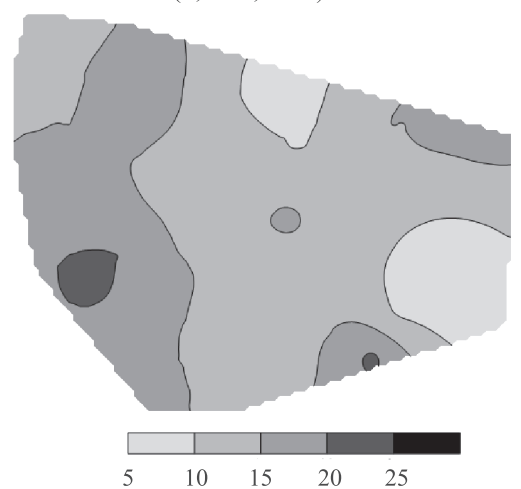

E

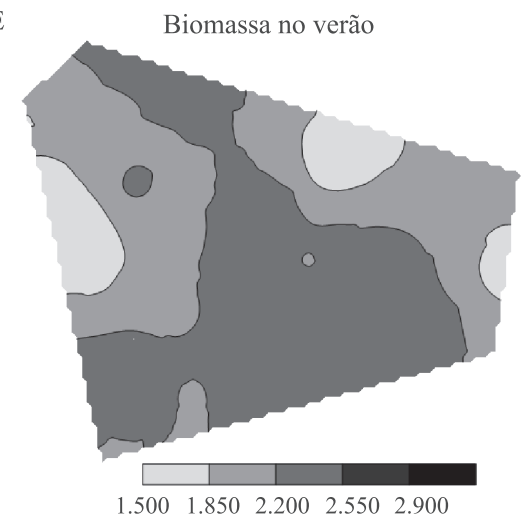

$\mathrm{C}$

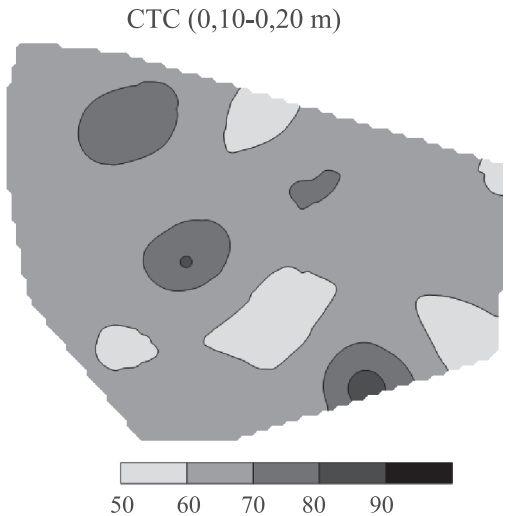

F

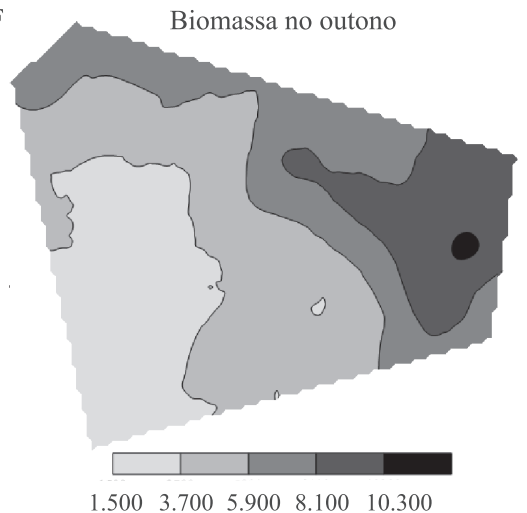

Figura 4. Mapas espacializados por krigagem ordinária dos atributos químicos do solo e da biomassa da pastagem: $\mathrm{A}$, $\mathrm{H}+\mathrm{Al}$ $\left(\mathrm{mmol}_{\mathrm{c}} \mathrm{dm}^{-3}\right) ; \mathrm{B}, \mathrm{Al}\left(\mathrm{mmol}_{\mathrm{c}} \mathrm{dm}^{-3}\right) ; \mathrm{C}, \mathrm{CTC}\left(\mathrm{mmol}_{\mathrm{c}} \mathrm{dm}^{-3}\right)$; D, saturação por bases (\%); E e F, biomassa (kg ha-1 de matéria seca) no verão e outono, respectivamente.

\section{Conclusões}

1. A análise espacial de atributos físicos do solo permite identificar áreas degradadas da pastagem.

2. Há dependência espacial nos mapas de $\mathrm{pH}, \mathrm{CTC}$, $\mathrm{V}$, e entre os mapas de matéria orgânica, $\mathrm{Ca}$ e $\mathrm{Mg}$.

3. A produção de biomassa é mais influenciada pelos atributos físicos do solo do que pelos químicos.

\section{Referências}

BONFIM, E.R.P.; PINTO, J.C.; SALVADOR, N.; MORAIS, A.R. de; ANDRADE, I.F. de; ALMEIDA, O.C. de. Efeito do tratamento físico associado à adubação em pastagem degradada de braquiária, nos teores de proteína bruta, fibra em detergente neutro e fibra em detergente ácido. Ciência e Agrotecnologia, v.27, p.912-920, 2003.

CAMARGO, O.A. de; MONIZ, A.C.; JORGE, J.A.; VALADARES, J.M.A.S. Métodos de análise química, mineralógica e física de solos do Instituto Agronômico de Campinas. Campinas: Instituto Agronômico de Campinas, 1986. 93p. (IAC. Boletim técnico, 106).
CEDDIA, M.B.; VIEIRA, S.R.; VILLELA, A.L.O.; MOTA, L. dos S.; ANJOS, L.H.C. dos; CARVALHO, D.F. de. Topography and spatial variability of soil physical properties. Scientia Agricola, v.66, p.338-352, 2009.

CLAESSEN, M.E.C. (Org.). Manual de métodos de análise de solo. 2.ed. rev. atual. Rio de Janeiro: Embrapa-CNPS, 1997. 212p. (Embrapa-CNPS. Documentos, 1).

DIAS-FILHO, M.B. Degradação de pastagens: processos, causas e estratégias de recuperação. 4.ed. Belém: Embrapa Amazônia Oriental, 2011. 215p.

FISHER, M.J.; BRAZ S.P.; DOS SANTOS, R.S.M.; URQUIAGA, S.; ALVES, B.J.R.; BODDEY, R.M. Another dimension to grazing systems: soil carbon. Tropical Grasslands, v.41, p.65-83, 2007.

GREGO, C.R.; COELHO, R.M.; VIEIRA, S.R. Critérios morfológicos e taxonômicos de Latossolo e Nitossolo validados por propriedades físicas mensuráveis analisadas em parte pela geoestatística. Revista Brasileira de Ciência do Solo, v.35, p.337-350, 2011.

HAYDOCK, K.P.; SHAW, N.H. El método de rendimento comparativo para estimar rendimento de matéria seca de pradarias. 
Australian Journal of Experimental Agriculture and Animal Husbandry, v.15, p.663-670, 1975.

LANZANOVA, M.E.; NICOLOSO, R. da S.; LOVATO, T.; ELTZ, F.L.F.; AMADO, T.J.C.; REINERT, D.J. Atributos físicos do solo em sistema de integração lavoura-pecuária sob plantio direto. Revista Brasileira de Ciência do Solo, v.31, p.1131-1140, 2007.

MIGUEL, F.R.M.; VIEIRA, S.R.; GREGO, C.R. Variabilidade espacial da infiltração de água em solo sob pastagem em função da intensidade de pisoteio. Pesquisa Agropecuária Brasileira, v.44, p.1513-1519, 2009.

MOREIRA, W.H.; BETIOLI JUNIOR, E.; PETEAN, L.P.; TORMENA, C.A.; ALVES, S.J.; COSTA, M.A.T.; FRANCO, H.H.S. Atributos físicos de um Latossolo Vermelho distroférrico em sistema de integração lavoura-pecuária. Revista Brasileira de Ciência do Solo, v.36, p.389-400, 2012.

NUMATA, L.; SOARES, J.V.; LEÔNIDAS, F.C. Comparação da fertilidade de solos em Rondônia com diferentes tempos de conversão de floresta em pastagem. Revista Brasileira de Ciência do Solo, v.26, p.949-955, 2002.

PERON, A.J.; EVANGELISTA, A.R. Degradação de pastagens em regiões de cerrado. Ciência e Agrotecnologia, v.28, p.655-661, 2004.

PIRES, B.S.; DIAS JUNIOR, M. de S.; ROCHA, W.W.; ARAUJO JUNIOR, C.F.; CARVALHO, R. de C.R. Modelos de capacidade de suporte de carga de um Latossolo Vermelho-amarelo sob diferentes usos e manejos. Revista Brasileira de Ciência do Solo, v.36, p.635-642, 2012.

SANTOS, R.S.M. dos; OLIVEIRA, I.P. de; MORAIS, R.F. de; URQUIAGA, S.C.; BODDEY, R.M.; ALVES, B.J.R. Componentes da parte aérea e raízes das pastagens de Brachiaria spp. em diferentes idades após a reforma, como indicadores de produtividade em ambiente de Cerrado. Pesquisa Agropecuária Tropical, v.37, p.121-124, 2007.

SÃO PAULO (Estado). Secretaria de Agricultura e Abastecimento. Coordenadoria de Assistência Técnica Integral. Instituto de Economia Agrícola. Levantamento censitário das unidades de produção agropecuária do Estado de São Paulo: LUPA 2007/08. São Paulo: SAA/CATI/IEA, 2008. Disponível em: <http://www. cati.sp.gov.br/projetolupa>. Acesso em: 03 set. 2012.
SILVA NETO, S.P. da; SANTOS, A.C. dos; LEITE, R.L. de L.; DIM, V.P.; NEVES NETO, D.N. das; CRUZ, R.S. da. Dependência espacial em levantamentos do estoque de carbono em áreas de pastagens de Brachiaria brizantha cv. Marandu. Acta Amazonica, v.42, p.547-556, 2012.

SOUZA, Z.M. de; MARQUES JÚNIOR, J.; PEREIRA, G.T.; MONTANARI, R. Otimização amostral de atributos de latossolos considerando aspectos solo-relevo. Ciência Rural, v.36, p.892-836, 2006.

STOLF, R. Teoria e teste experimental de fórmulas de transformação dos dados de penetrômetro de impacto em resistência do solo. Revista Brasileira de Ciência do Solo, v.15, p.229-235, 1991.

TAVARES FILHO, J.; RIBON,A.A. Resistência do soloà penetração em resposta ao número de amostras e tipo de amostragem. Revista Brasileira de Ciência do Solo, v.32, p.487-494, 2008.

VIDAL-VÁZQUEZ, E.; PAZ-FERREIRO, J.; VIEIRA, S.R.; TOPP, G.C.; MIRANDA, J.G.V.; PAZ GONZALEZ, A. Fractal description of the spatial and temporal variability of soil water content across an agricultural field. Soil Science, v.177, p.131-138, 2012.

VIEIRA, S.R. Geoestatística em estudos de variabilidade espacial do solo. In: NOVAIS, R.F. de; ALVAREZ V., V.H.; SCHAEFER, C.E.G.R. (Ed.). Tópicos em ciência do solo. Viçosa: Sociedade Brasileira de Ciência do Solo, 2000. p.1-53.

VIEIRA, S.R. Permeâmetro: novo aliado na avaliação de manejo do solo. O Agronômico, v.50, p.32-33, 1998.

VIEIRA, S.R.; DECHEN, S.C.F. Spatial variability studies in São Paulo, Brazil along the last twenty five years. Bragantia, v.69, p.53-66, 2010.

VIEIRA, S.R.; MILLETE, J.A.; TOPP, G.C.; REYNOLDS, W.D. Handbook for geostatistical analysis of variability in soil and meteorological parameters. In: ALVAREZ V.V.H.; SCHAEFER, C.E.G.R.; BARROS, N.F. de; MELLO, J.W.V. de; COSTA, L.M. da. (Ed.). Tópicos em ciência do solo. Viçosa: Sociedade Brasileira de Ciência do Solo, 2002. v.2, p.1-45.

VIEIRA, S.R.; XAVIER, M.A.; GREGO, C.R. Aplicações de geoestatística em pesquisa com cana-de-açúcar. In: DINARDO MIRANDA, L.L.; VASCONCELOS, A.C.M. de; LANDELL, M.G. de A. (Ed.). Cana-de-açúcar. Campinas: Instituto Agronômico, 2008. p.839-852.

Recebido em $1^{\circ}$ de setembro de 2011 e aprovado em 9 de agosto de 2012 